\title{
Energy exponents and corrections to scaling in Ising spin glasses
}

\author{
J.-P. Bouchaud, ${ }^{1}$ F. Krzakala, ${ }^{2,3}$ and O. C. Martin ${ }^{1,2}$ \\ ${ }^{1}$ Service de Physique de l'État Condensé, Orme des Merisiers - CEA Saclay, 91191 Gif sur Yvette Cedex, France. \\ ${ }^{2}$ Laboratoire de Physique Théorique et Modèles Statistiques, \\ bât. 100, Université Paris-Sud, F-91405 Orsay, France. \\ ${ }^{3}$ Dipartimento di Fisica, INFM, SMC, Università di Roma La Sapienza P. A. Moro 2, 00185 Rome, Italy.
}

(Dated: July 3, 2018)

\begin{abstract}
We study the probability distribution $P(E)$ of the ground state energy $E$ in various Ising spin glasses. In most models, $P(E)$ seems to become Gaussian with a variance growing as the system's volume $V$. Exceptions include the Sherrington-Kirkpatrick model (where the variance grows more slowly, perhaps as the square root of the volume), and mean field diluted spin glasses having $\pm J$ couplings. We also find that the corrections to the extensive part of the disorder averaged energy grow as a power of the system size; for finite dimensional lattices, this exponent is equal, within numerical precision, to the domain-wall exponent $\theta_{D W}$. We also show how a systematic expansion of $\theta_{D W}$ in powers of $e^{-d}$ can be obtained for Migdal-Kadanoff lattices. Some physical arguments are given to rationalize our findings.
\end{abstract}

PACS numbers: PACS Numbers : 75.50.Lk, 05.50.+q

\section{INTRODUCTION}

Exponents and corrections to scaling play a central role in disordered systems. Consider for instance the dependence of thermodynamic quantities on the size $L$ of a system when $L \rightarrow \infty$. In paramagnetic systems, correlations are short range and thus bulk properties converge rapidly to their thermodynamic limits. On the contrary, in systems such as spin glasses below $T_{c}$, correlations are long range and so finite size corrections are "large". In practice, that means that even if one takes periodic boundary conditions, the disorder average of an intensive quantity converges to its thermodynamic limit slowly: finite size corrections go to zero as an inverse power of $L$. This slow convergence is to be contrasted with paramagnetic systems where finite size corrections in disorder averages are exponentially small. One can also consider the sample-tosample fluctuations of thermodynamic quantities. These fluctuations scale in the thermodynamic limit; an interesting open question is their limiting distribution, if any.

In this work we consider these issues in the context of Ising spin glasses [1, 2] at zero temperature and focus on the ground state energy. Given a distribution of disorder realisations (each being represented by $J$ ), the extensive ground state energy $E_{J}$ is a random variable. Denote by $N$ the number of spins in the system; $N=L^{d}$ for a $d$-dimensional hyper-cubic lattice. We are interested in the probability distribution $P_{N}\left(E_{J}\right)$ and in how the connected moments (cumulants) of this distribution depend on $N$ (or $L$ ). Denoting disorder averages by an overline, we have

$$
\overline{E_{J}(L)}=e_{0} L^{d}+e_{1} L^{\Theta_{s}}+\cdots
$$

In this expression, $\Theta_{s}$ is what we call the shift exponent. To leading order, the energy scales with the volume, while $\Theta_{s}$ gives the leading correction to scaling. The justification for this notation will become clear later; in the mean time, we should compare to the usual notation involving the correction to scaling exponent $\omega$; indeed, in the spin glass phase, finite size corrections of intensive quantities decay as a power of $L$ :

$$
\overline{E_{J}(L) / L^{d}}=e_{0}+e_{1} L^{-\omega}+\cdots
$$

and so $\omega=d-\Theta_{s}$.

We will also look at the width of $P_{N}$ which gives the scaling of the instance to instance fluctuations:

$$
\left[\overline{E_{J}^{2}(L)}-{\overline{E_{J}(L)}}^{2}\right]^{1 / 2}=\sigma_{0} L^{\Theta_{f}}+\cdots
$$

$\Theta_{f}$ being the fluctuation exponent. Naturally, one may extend these types of expansions to any cumulant of $P_{N}$, a central question being whether $P_{N}$ has a limiting shape as $N \rightarrow \infty$. One motivation for this is the fact that the ground state energy is an extreme statistic: there are $2^{N}$ configurations of spins $\left(S_{i}= \pm 1\right)$ and one is interested in the one of minimum energy. (Note that these $2^{N}$ random energies are correlated.) Little is known about such statistics except in a few solvable cases [3, 4]. The purpose of this work is to determine $P_{N}\left(E_{J}\right)$ numerically for a variety of spin glass models. From our measurements we extract $\Theta_{s}, \Theta_{f}$, etc... and compare these results to theory.

The outline of this paper is as follows. We first consider expectations arising from analogies with other systems as well as some known results. In section III we describe the different types of spin glasses used in this study. Then we give in section IV our estimates of the fluctuation exponent $\Theta_{f}$. This is followed by a study of the probability distribution of the ground state energy in section $\square$ for most models we find that it becomes Gaussian in the large system size limit. Then in section VI we present our results for the shift exponent $\Theta_{s}$. For all of these studies, we compare the $d$-dimensional case to the theoretical predictions. In section VII we show that these exponents sometimes depend on the distribution of 
the disorder variables (the spin-spin couplings). Finally in section $\nabla I I I$ we discuss and conclude this work. Some details of the analytic computations are given in the appendix.

\section{CLUES FROM THEORY}

What values should be expected for the two exponents $\Theta_{s}$ and $\Theta_{f}$ ? Suppose we start with the random energy model (REM) [5] as a guide. Its ground state energy $E_{J}$ has a Gumbel distribution in the large $N$ limit [6] with a variance of $O(1)$. Also, the disorder averaged ground state energy grows as $e_{0} N+O(\ln N)$ at large $N$. These properties show that $\Theta_{s}=\Theta_{f}=0$ and thus in the REM finite size corrections are "small", though clearly much larger than in a paramagnetic system.

Another model that can guide us is the directed polymer in a random medium (DPRM) 7]. The problem on a tree is very similar to the REM, although subtle differences appear [4]. The two dimensional case (with one space and one "time" dimension) can also be solved in much detail. In these cases, perhaps surprisingly, a single exponent $\theta$ describes the scaling of three a priori unrelated quantities: (1) the corrections to scaling of the disorder averaged ground state energy $E_{J} ;(2)$ the size of sample-to-sample fluctuations of $E_{J} ;(3)$ the typical excitation energy of the lowest "system-size" excitation, that is an excitation that is macroscopically different from the ground state. These properties lead to the remarkable relation $\Theta_{s}=\Theta_{f}=\theta$. The DPRM is thus described by a "one-parameter" scaling theory.

It is plausible that an extension of the DPRM scaling theory may apply to Ising spin glasses. At the heart of such a theory, initiated by McMillan [8], developped by Bray and Moore [9] and extended by Fisher and Huse [10], is the exponent $\theta_{D W}$. This exponent is analogous to the $\theta$ of the DPRM, and gives the scaling of domain wall energies, $E_{D W} \simeq \Upsilon L^{\theta_{D W}}$; numerical estimates give $\theta_{D W} \approx 0.20$ in $d=3$ and $\theta_{D W} \approx-0.28$ in $d=2$. (More generally, a number of other $\theta$ exponents have been introduced for spin glasses; they are all associated with excitation energies; this is to be contrasted with our $\Theta$ exponents that are associated with ground state energies.) If we follow the correspondence with the DPRM, we expect that $\Theta_{s}=\theta_{D W}$, justifying our use of a "theta" notation for $\Theta_{s}$. Physically, this equality corresponds to the fact that these systems are sensitive to boundary conditions; for some samples, these conditions are such that a "domain wall" must be present in the ground state. However the analogy with the DPRM certainly breaks down for $\Theta_{f}$ : for any short range spin glass in dimension $d$, Wehr and Aizenman [1] proved that $\Theta_{f}=d / 2$. This shows that the REM and the DPRM are not good guides for finite dimensional spin glasses.

To have more realistic theoretical predictions, and in particular to preserve $\Theta_{f}=d / 2$, it seems necessary to work with models having a microscopic Hamiltonian de- fined over configurations of $N$ spins. One approach is to use hierarchical (Migdal-Kadanoff) lattices; there, analytical computations as well as powerful numerical methods are possible. We shall also consider mean field spin glasses where spins are coupled amongst one-another at random so there is no geometry to speak of. The $P_{N}\left(E_{J}\right)$ in such models can be referred to as the mean field prediction. Strangely enough, little is know about these systems so we will have to determine their behavior numerically. In some cases these models lead to surprizes as we shall soon see.

\section{MODELS AND METHODS}

We focus on three families of Ising spin glass models so that the effects of geometry and dimension are apparent. The Hamiltonian for these models is

$$
H=-\sum_{<i j>} J_{i j} S_{i} S_{j}
$$

where $S_{i}= \pm 1$ and the $J_{i j}$ are quenched Gaussian random variables of zero mean and variance 1 (except in the case of the Sherrington-Kirkpatrick model which we discuss later). The sum $\sum_{\langle i j\rangle}$ is over nearest neighbor spins on a given graph having $N$ nodes; the different models we consider vary simply by the nature of that graph.

Our first family of models are of the Edwards-Anderson (EA) type 12]: the graphs are square or cubic lattices of linear size $L$ (thus $N=L^{d}$ when the dimension is $d$ ), and the edges connect nearest neighbors only; we take periodic boundary conditions in all directions.

Our second family of models come from the MigdalKadanoff (MK) approach [13] where one performs a bond-moving real-space renormalization group. This procedure effectively amounts to computing quantities on hierarchical (MK) lattices defined by an iteration process (see Fig. 1). The iteration takes one bond (that is an edge of the current graph) into $b$ paths, each made of 2 segments (that is edges); if $r$ is the iteration number (beginning with $r=0$ ), the "linear" lattice size $L$ grows as $2^{r}$ and the volume (actually the number of edges and thus the number of terms contributing to the energy) grows as $(2 b)^{r}$. When using $\ell$ segments instead of 2 in each path, we have

$$
L=\ell^{r} \text { and } N \simeq(\ell b)^{r}
$$

so that the dimension is $d=\ln N / \ln L=1+\ln b / \ln \ell$. The usual choice to obtain $d=3$ is $\ell=2$ and $b=4$, while $\ell=b=2$ corresponds to $d=2$ as in Fig. 1]

Our third family of models come from mean field, and here we have considered two types of graphs. First, we use complete graphs where all vertices are connected, corresponding to the Sherrington-Kirkpatrick (SK) model 14]. To have an extensive energy, one takes the variance of the $J_{i j}$ to be $1 / N$. Second, we also use 


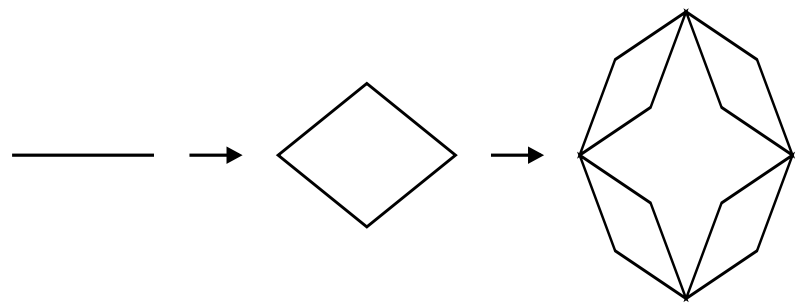

FIG. 1: Construction of a $d=2 \mathrm{MK}$ hierarchical lattice.

diluted models for which the connectivity is fixed and identical for all the vertices of the graph [15]. The disorder ensemble then consists of the uniform distribution over all graphs satisfying that constraint in addition to the disorder ensemble in the $J_{i j}$. Such an ensemble can be used to "model" the Euclidean case by setting the coordination to that of the lattice of interest. Thus to model the $d$-dimensional EA model on the hypercubic lattice, we set the coordination to $2 d$.

For these three families of spin glass models, we will determine the distribution of the ground state energies. The hierarchical lattices allow one to write a recursion for $P_{N}\left(E_{J}\right)$; because of that, there is no need to sample the disorder variables, we can perform the disorder average exactly. On the contrary, for the EA and the mean field cases, we must compute the actual ground state energies for a large number of disorder samples. For that we rely on a previously tested [16] algorithmic procedure where given enough computational ressources, the ground state is obtained with a very high probability for both $\mathrm{Eu}-$ clidean lattices and for random graphs as long as $N$ is not too large. For our runs, we used several months of CPU time on Pentium III personal computers running at $666 \mathrm{MHz}$. With this amount of CPU, we obtained high statistics for lattices of sizes up to $L=10$ (in both $d=2$ and 3) and for mean field graphs with $N$ up to 300 .

\section{SAMPLE TO SAMPLE FLUCTUATIONS AND THE EXPONENT $\Theta_{f}$}

\section{A. Migdal-Kadanoff lattices}

Let us begin with the Migdal-Kadanoff approach for which the important prediction $\Theta_{f}=d / 2$ can be derived. To understand how this relation comes about on the hierarchical lattices, we construct these by "aggregation", i.e., by recursively (and hierarchically) joining sub-lattices together. (This procedure is to be distinguished from the top-down iteration used in Fig. 10 We work with the distribution of ground state energies $E_{p}$ (respectively $E_{a}$ ) subject to fixed boundary conditions, the spins on the ends of the lattice being forced to be parallel (respectively antiparallel.) Let $\sigma^{2}$ be the variance of the ground state energy at some level of the hierarchical construction. To go to the next level for which $L$ will be $\ell$ times larger, first find the ground state energy in one of the $b$ paths. Clearly, if we take the ground state configuration in each of the segments of that path, we will have built the ground state for unconstrained end spins. If the result does not give the imposed values for the end spins (parallel or antiparallel), one must add a "correction" term equal to the smallest domain wall energy of the $\ell$ segments of that path. Thus the energy of one path is the sum of the ground state energies of the $\ell$ segments, plus one domain wall energy half of the time. Second, we add up the independent contributions from the $b$ different paths, leading to $E_{p}$ and $E_{a}$ at the new level. The ground state energy is then simply $\min \left(E_{p}, E_{a}\right)$. If we neglect the "correction" term, then $\sigma^{2}$ at this new level is just $\ell b$ times larger than at the previous level. At large $b$, the correction is in fact small and so it can be neglected. From one level to the next, the volume grows by a factor $\ell b$, just as $\sigma^{2}$ does, so the variance is linear in the lattice volume and thus $\Theta_{f}=d / 2$. From our numerical study of these hierarchical lattices, we find that this relation holds also for small $b$ and for both $\ell=2$ and $\ell=3$. (We did not test for larger $\ell$.)

As mentioned in the introduction, the Wehr-Aizenman theorem [11] shows that $\Theta_{f}=d / 2$ in finite dimensional spin glasses. It is rather conforting that the MK approach also leads to this result, sustaining the belief that it is a useful guide for real (finite dimensional) spin glasses.

\section{B. Mean field models}

Next, consider the mean field prediction for $\Theta_{f}$. Since there is no geometry in our mean field family of models, we identify $L$ with $N^{1 / d}$ and thus

$$
\left[\overline{E_{J}^{2}(N)}-{\overline{E_{J}(N)}}^{2}\right]^{1 / 2}=\sigma_{0} N^{\Theta_{f} / d}+\cdots
$$

The issue here is whether mean field also predicts $\Theta_{f} / d=$ $1 / 2$ as it should if one believes that this approach correctly describes the large dimensional limit of real spin glasses. To find out, we have performed ground state computations on thousands of samples of the SK model and of fixed connectivity spin glasses, and have extracted for each ensemble its associated $\Theta_{f} / d$.

First, consider the fixed connectivity spin glasses with the connectivities, $z=3,4,6$ and 10 . To estimate $\Theta_{f} / d$, we determine when the root mean square deviation (RMS) of the ground state energy divided by $N^{\Theta_{f} / d}$ becomes flat as a function of $N$. (Our runs were performed for $50 \leq N \leq 300$.) This leads to $\Theta_{f} / d \approx 0.5$ for $z=3, \Theta_{f} / d \approx 0.49$ for $z=4, \Theta_{f} / d \approx 0.48$ for $z=6$, and $\Theta_{f} / d \approx 0.44$ for $z=10$. The drift we observe in $\Theta_{f} / d$ is most probably an artefact of our procedure, and simply corresponds to the fact that the corrections to the scaling in Eq. 6] are important in our data, especially at large $z$. To get better estimates of $\Theta_{f} / d$, we would need to control these corrections to scaling but our data are not sufficiently precise for that. Nevertheless, it seems very plausible that $\Theta_{f} / d=0.5$ in all the fixed connectivity 


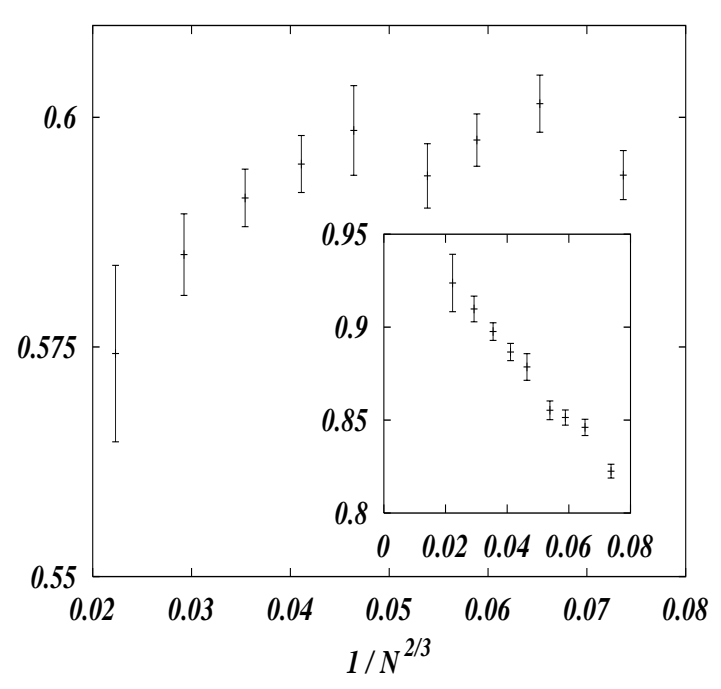

FIG. 2: Scaled root mean square deviation (RMS) of the ground state energy in the SK model, displayed as a function of $N^{-2 / 3}$. Main figure: RMS divided by $N^{1 / 4}$; inset: RMS divided by $N^{1 / 6}$.

models. In direct analogy to what was stated previously, we can also say that this result sustains the belief that mean field models provide a useful guide to real (finite dimensional) spin glasses.

Let us now continue and consider the limit of infinite connectivity, i.e., the SK model. Since there are $O\left(N^{2}\right)$ terms contributing to the Hamiltonian, a very simple minded guess would give $\Theta_{f} / d=1$ and thus larger fluctuations than in the other models. However the opposite happens, revealing that the scaling in the SK is quite subtle. One can get some clues from Kondor's analytic study [17 performed just below the critical temperature $T_{c}$. In particular, his results have been interpreted by Crisanti et al. [18] who argued that the free energy fluctuations should scale as $N^{1 / 6}$, which is very small compared to the naïve estimate. A different estimate was recently proposed by Aspelmeier et al. [19] who argued that free energy fluctuations should scale as $N^{1 / 4}$. Our concern here is the ground state energy; although there are no analytical calculations, it is plausible that the exponent for energy fluctuations at $T=0$ is the same as at that for free energy fluctuations at $0<T<T_{c}$. (Such an extrapolation to $T=0$ is known to apply to the DPRM where the exponent of the free energy fluctuations at $T>0$ is equal to the one of the ground state energy fluctuations.) In that case, we would have $\Theta_{f} / d=1 / 6$ according to some authors and $\Theta_{f} / d=1 / 4$ according to others.

What do the numerical estimates tell us about this question? We are aware of a study by Cabasino et al. 20] who showed beyond any reasonable doubt that $\Theta_{f} / d<$ 0.5 ; in fact their best fit gives $\Theta_{f} / d \approx 0.28$. When we consider our data, motivated by Eq. [6 we find that the ratio of the left and right hand sides is compatible with a constant when we use $\Theta_{f} \approx 0.25$ and $40 \leq N \leq 150$, but the two points at $N=200$ and 300 are then below the others as show in Fig. 22 It is difficult to extract an error bar on the value of this exponent, and most likely the terms dropped in Eq. [6]are important just as was the case in the fixed connectivity models. In fact, here the difficulty is even more acute as the standard deviation increases only very slowly with $N$. Nevertheless, let us fit the standard deviation to a pure power; then using a $\chi^{2}$ analysis we obtain $\Theta_{f} / d=0.24 \pm 0.005$ which is very close to the conjectured $1 / 4$ value, a result further supported by other numerical work 21]. Note however that throughout all this paper, the error bars given are statistical only... In our fit, there are 7 degrees of freedom and the resulting $\chi^{2} \approx 9.5$ is not bad; nevertheless, a critical examination of the figure leads one to conclude that the actual uncertainty on $\Theta_{f} / d$ is certainly much greater than 0.005 . Note that this estimate is a bit lower that that of [20] and is many standard deviations away from the conjecture $\Theta_{f} / d=1 / 6$. However, if we impose $\Theta_{f} / d=1 / 6$, the data is reasonably straight as a function of $N^{-2 / 3}$ as shown in the inset of Fig. 2] Because of this "good" behavior, we cannot rule out $\Theta_{f} / d=1 / 6$.

Given that in the dilute spin glasses we expect $\Theta_{f} / d=$ 0.5 , how does one recover the SK case as $z \rightarrow \infty$ ? Begin by recalling that, in the SK model, the variance of the $J_{i j}$ is taken to be $1 / N$ to ensure that the ground state energy is extensive. To maintain this property in the diluted spin glasses, we must divide the $J_{i j}$ by $\sqrt{z}$. The fluctuations for large $N$ and $z$ in these modified spin glasses scale as

$$
\left[\overline{E_{J}^{2}(N)}-{\overline{E_{J}(N)}}^{2}\right]^{1 / 2}=\sigma_{0}(z) N^{\Theta_{f} / d} / \sqrt{z}+\cdots
$$

with $\Theta_{f} / d=1 / 2$ from what we saw previously. The SK limit corresponds to setting $z=N$, so we see that smooth large $N$ and $z$ limits require $\sigma_{0}(z) \simeq z^{\mu}$ where $\mu$ is equal to the SK value of $\Theta_{f} / d$. We saw that it was difficult to obtain $\Theta_{f} / d$ in the fixed connectivity models, but obtaining the prefactor $\sigma_{0}(z)$ is even more difficult. Nevertheless, we have extrapolated our data for the root mean square deviation divided by $N^{0.5}$ in the different finite connectivity models. This leads to the estimates $\sigma_{0}(z=3) \approx 0.67, \sigma_{0}(z=4) \approx 0.70, \sigma_{0}(z=6) \approx 0.73$, and $\sigma_{0}(z=10) \approx 0.76$. This growth is very slow; it is compatible with the value $\mu=1 / 6$ but much less with the value $\mu=0.25$. Given the large uncertainties in our extrapolations however, this has to be considered as only very indirect evidence in favor of $\Theta_{f} / d=1 / 6$.

We conclude that understanding the non-trivial size dependence of the fluctuations of the ground state energy in the SK model remains quite a challenge. Let us propose here a simple argument suggesting that $\Theta_{f} / d=1 / 4$. Imagine that one changes slowly all the couplings $J_{i j}=$ $J_{i j}^{0} / \sqrt{N}: J_{i j}^{0} \rightarrow J_{i j}^{0}+\delta J_{i j}^{0}$, where the order of magnitude of $\delta J_{i j}^{0}$ is denoted by $\varepsilon$. If $\varepsilon$ is infinitesimal, the ground state remains the same. Up to what value of $\varepsilon$ will this be true? The change of local field induced by the change of couplings for a fixed configuration $\left\{S_{i}^{*}\right\}$ of the spins is of order $\sum_{j} \delta J_{i j}^{0} S_{j}^{*} / \sqrt{N} \sim \varepsilon$. Using the fact that the 
local field distribution vanishes linearly for small fields in the SK model, it is easy to show that the smallest local field is of $O(1 / \sqrt{N})$. Therefore, the first value of $\varepsilon$ that will trigger a change of the ground state is $\varepsilon^{*} \sim 1 / \sqrt{N}$. Flipping the spin with this low local field should lead to a cascade of flips that lowers the energy and thus to a new ground state that is "substantially" different from the starting one. Furthermore, the variation in the ground state energy when going from $\varepsilon=0$ to $\varepsilon=\varepsilon^{*}$ is probably $O(1)$; indeed, there are excited states of energy $O(1)$ above the ground state, and one therefore expects level crossings to occur when $\varepsilon \sim 1 / \sqrt{N}$. Finally, in order to scan the whole distribution of $J_{i j}^{0}$ 's, one needs $\varepsilon \sim 1$. Using this range, the ground state will change $1 / \varepsilon^{*} \sim N^{0.5}$ times. Since between each level crossing, the ground state energy randomly changes by an amount $O(1)$, the total expected fluctuation of the ground state energy will be $O\left(N^{1 / 4}\right)$, so that $\Theta_{f} / d=1 / 4$. More analytical work is obviously needed to confirm this speculative result, but note that at the heart of our argument lies the fact that the ground state of the SK model is particularly fragile: a relative change of order $1 / \sqrt{N}$ of the disorder is enough to substantially change the ground state.

\section{Edwards-Anderson models}

In the case of the finite dimensional lattices, we know that $\Theta_{f}=d / 2$ holds exactly because of the WehrAizenman theorem [1], and recently Aspelmeier and Moore 22] found that this relation holds within replica theory. In spite of these theoretical results, it is instructive to see how this equality transpires numerically. We thus follow the procedure used in the fixed connectivity models where we tested for when the rescaled RMS became size independent. In $d=3$, the rescaled data show no obvious trend when $1.49 \leq \Theta_{f} \leq 1.60$ while in $d=2$ the corresponding range is $1.00 \leq \Theta_{f} \leq 1.02$. In particular, in Fig. 3 we show these ratios when $\Theta_{f}$ is set to $d / 2$ (the data displayed are from the models with Gaussian $J_{i j} \mathrm{~s}$.)

In principle, it would be interesting to find the corrections to this scaling law. In a renormalization group picture, corrections go as inverse powers of $L$. Furthermore, if one believes that the exponent $\Theta_{s}$ gives the leading corrections to scaling for all extensive quantities, then those for the variance of $E_{J}$ should go as $L^{\Theta_{s}}$. To consider this possibility, we set $\Theta_{f}=d / 2$ and then ask when our data for the rescaled RMS follow approximately a straight line when plotted as a function of $L^{-\omega}$ with the expectation that $\omega=d-\Theta_{s}$. In $d=3$, we find $\omega \geq 1.5$, to be compared with the "theoretical" value $\omega \approx 2.8$ (cf. section (VI). Since $\Theta_{s}$ is small and may in fact be zero, we have performed the analysis for $\omega=3$; using the intercept of the curve with the $y$ axis, we find that the RMS of $E_{J}$ grows as $0.765 L^{3 / 2}$. In $d=2$, the rescaled data is too flat and so in practice we cannot give any sensible estimate of $\omega$. But we can follow the $d=3$ procedure,

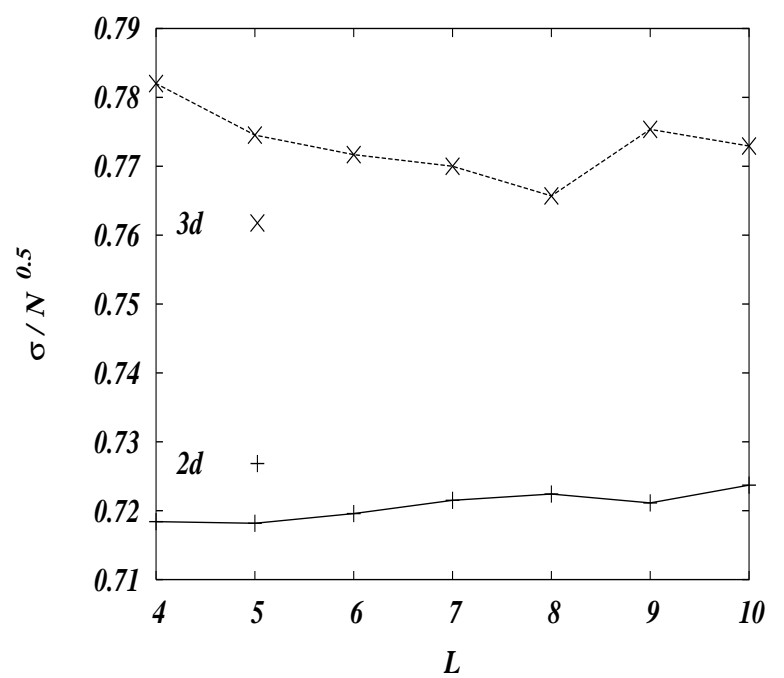

FIG. 3: Standard deviation of the ground state energy divided by the square root of the volume for the EA model in $d=2$ and 3 , Gaussian $J_{i j}$ s.

setting this time $\omega=2\left(\Theta_{s}\right.$ is also relatively close to zero in $d=2$ ); the corresponding fits give that that the RMS of $E_{J}$ grows as $0.725 \mathrm{~L}$. The moral of this story is that corrections to scaling are in general very difficult to determine, even if the leading scaling law is known exactly. Nevertheless, it seems that the same $\omega$ may very well describe the dominant corrections to scaling of many observables, as expected from the renormalization group picture.

To put these last numbers in perspective, consider the Mattis model where the couplings are gauge transformed from a ferromagnet having $J_{i j}$ chosen randomly on the positive side of a Gaussian. The total ground state energy of such a system is the sum of all these couplings and thus has the expectation value $d N \sqrt{2 / \pi}$ on the hypercubic lattice of dimension $d$. On the other hand its variance is $d N[1-2 / \pi]$. Thus in this Mattis model, the RMS of the ground state energy grows as $1.04 L^{3 / 2}$ in $d=3$, and as $0.852 L$ in $d=2$. As expected, the Mattis model has larger absolute fluctuations than the EA model. It is also appropriate to compare the relative fluctuations $\sigma_{r}$, that is the RMS of the ground state energy divided by its mean. For the Mattis model, we find $\sigma_{r}=0.434 / L^{3 / 2}$ in $d=3$ and $\sigma_{r}=0.534 / L$ in $d=2$. These should be compared to the values we find in the EA model: $\sigma_{r}=$ $0.450 / L^{3 / 2}$ in $d=3$ and $\sigma_{r}=0.551 / L$ in $d=2$. This shows that the relative fluctuations are slightly smaller in the Mattis model than in the EA model. Although this is in line with what frustration should do, note that the size of the effect is about $3 \%$ which is very very small.

To summarize our study of $\Theta_{f}$ for the different spin glass models, we have found that all the models considered seem to satisfy $\Theta_{f}=d / 2$. The notable exception is the SK model for which we made the case that $\Theta_{f}=d / 4$ was likely but $\Theta_{f}=d / 6$ was also possible; no matter 
what, $\Theta_{f}=d / 2$ is excluded, showing that the $N \rightarrow \infty$ and $d \rightarrow \infty$ limits do not commute. In all other cases, the variance of the ground state energy grows linearly with the system's volume; this is the scaling expected from a central limit theorem behavior when the different terms contributing to the ground state energy are independent. Our results thus tell us that these terms are only weakly correlated. This feature is completely missed by both the REM and the DPRM; although a one-parameter scaling picture applies to those two models, it cannot apply to spin glasses. Note that this central limit behavior suggests that $P_{N}\left(E_{J}\right)$ tends to a Gaussian; we now turn to see whether this is the case.

\section{PROBABILITY DISTRIBUTION OF GROUND STATE ENERGIES}

If the central limit theorem (CTL) were applicable, not only would we have $\Theta_{f}=d / 2$ for the scaling of the fluctuations, but also the shape of $P_{N}\left(E_{J}\right)$ would become Gaussian at large $L$. This behavior indeed arises for the MK lattices, both analytically at large $b$ and numerically for all $b$. (In our numerical study, we find that the skewness and kurtosis $P_{N}\left(E_{J}\right)$ decrease fast towards zero as $L$ grows.) Obviously, the terms contributing to the ground state energy are not independent but their correlations are not strong enough to prevent a CLT large $L$ scaling.

The question we address here is whether this simple behavior also holds in the other models. Let us begin with the mean field case. For the fixed connectivity mean field graphs, our data for the skewness and kurtosis of $P_{N}\left(E_{J}\right)$ decrease in magnitude as $N$ increases; this decrease is compatible with an extrapolation to zero as $N \rightarrow \infty$ as illustrated in Fig. 团 (These quantities are difficult to measure to high precision, so this should be considered as only suggestive of a Gaussian limit for $P_{N}\left(E_{J}\right)$.) The SK model however is clearly in a different class: not only does it have $\Theta_{f} \neq d / 2$ but also its $P_{N}\left(E_{J}\right)$ is not Gaussian. In particular, its skewness at large $N$ stabilizes around $-0.43 \pm 0.02$, while its kurtosis stabilizes around $0.40 \pm 0.03$. It is instructive to compare this to the values predicted by the REM model where $P\left(X_{J}\right)$ is a Gumbel distribution: there, the skewness is -1.139 while the kurtosis is exactly 2.4. Our estimates do not agree either with the values from the Fisher or Weibull universality classes: we thus conclude that the SK ground state energy distribution does not belong to one of the known universality classes of extreme statistics $[\underline{6}$.

Finally we come to the EA lattices. Given that the MK and diluted mean-field graphs lead to the same conclusion, it will come as no surprise that our data for the EA lattices are also compatible with a Gaussian limiting shape for $P_{N}\left(E_{J}\right)$. Note that this is expected though not proven from the work of Wehr and Aizenman 11], while it does follow from replica theory calculations 22 . An examination of the skewness and kurtosis of $P_{N}\left(E_{J}\right)$ shows that they decay with system size, both in $d=2$

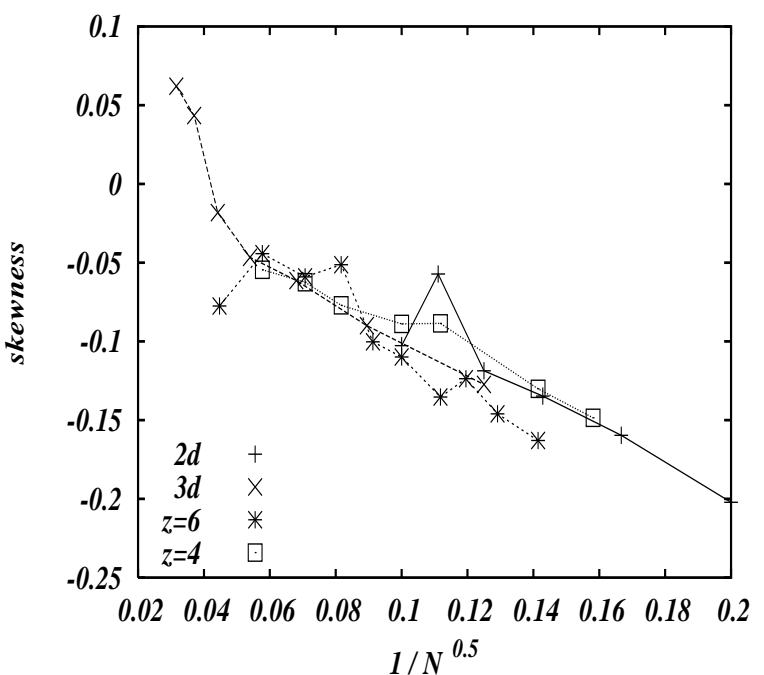

FIG. 4: Skewness of the ground state energy distribution for mean field and EA models with Gaussian $J_{i j}$ s.

and $d=3$. Although our measurements lack precision when the number of spins is large, the extrapolations suggest zero limiting values as $L \rightarrow \infty$ as one can see

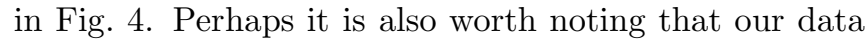
are quantitatively similar in the different models; for instance, for $L=8$ and $d=3$, we find the skewness to be -0.18 in EA and -0.21 in MK.

The overall situation indicates that $P_{N}\left(E_{J}\right)$ is Gaussian. Furthermore we checked whether the convergence to this Gaussian follows the central limit theorem law. Indeed, that law predicts for instance that the skewness scales as $N^{-1 / 2}$; thus we have plotted in Fig. 团 the skewness for the different models as a function of that scale. The data is completely compatible with a linear convergence to zero, confirming the CLT scaling. Only in the case of the SK model are the variables contributing to the ground state energy sufficiently correlated to prevent a central limit theorem behavior. A physical interpretation of this is that as soon as there is a kind of locality which allows one to decompose the sample into quasiindependent subsystems, the central limit theorem behavior will appear, leading to $\Theta_{f}=d / 2$ and a Gaussian $P_{N}\left(E_{J}\right)$. Of course, it is not clear why this should apply to the diluted mean field graphs.

\section{THE SHIFT EXPONENT $\Theta_{s}$}

\section{A. Migdal-Kadanoff lattices}

We now move on and study the finite size corrections to the mean energy density. Following Eq. 10 the mean excess of the (extensive) ground state energy is expected to scale as $L^{\Theta_{s}}$. To have an idea of what this exponent should be, it is again most useful to begin with the hierarchical lattices. The important prediction of that 


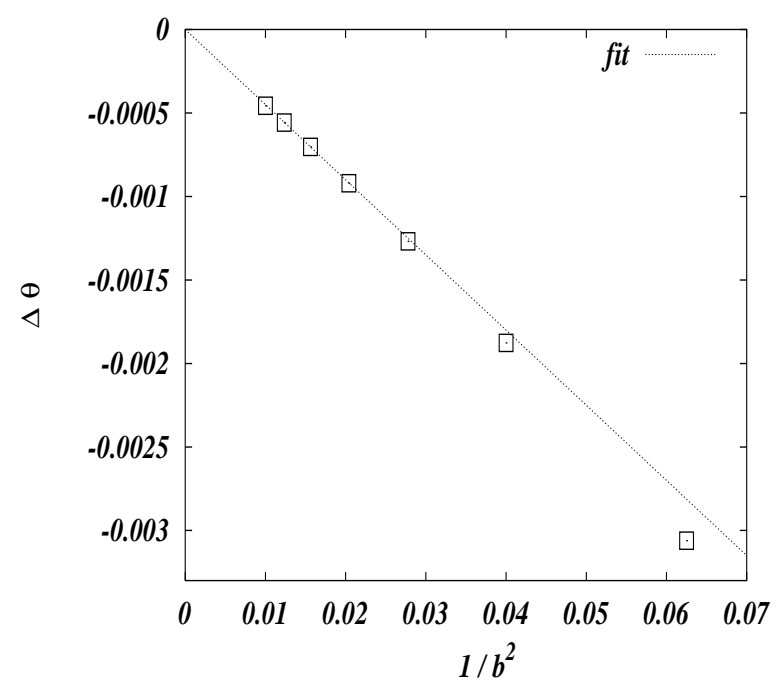

FIG. 5: Difference between $\theta_{D W}$ and the first three terms in the series of Eq. 8 as a function of $1 / b^{2}$ where $b$ is the parameter of the Migdal-Kadanoff lattices. The line is $-0.045 / b^{2}$.

approach is that $\Theta_{s}=\theta_{D W}$ where $\theta_{D W}$ is the domain wall exponent. To see why this is so, reconsider the evolution equation for the energies $E_{p}$ and $E_{a}$ as one applies the recursion. First, along a given path, the energy is the sum of the ground state energies of each of its $\ell$ segments, the sum being sometimes corrected by the energy of the domain wall of the weakest segment in order to satisfy the boundary conditions. This correction shifts the path's energy by $O\left(L^{\theta_{D W}}\right)$. Second, adding the energies of the different paths does not change the scale of the shifts, so necessarily $\Theta_{s}=\theta_{D W}$. Naturally, we have confirmed this relation numerically for different values of $\ell$ and $b$ by performing fits to moments of $P\left(E_{p}, E_{a}\right)$ (determined with no statistical error for these hierarchical lattices) from which we extracted estimates for $\Theta_{s}$ and $\theta_{D W}$

When $b$ is large, we can derive the analytical expression for $\theta_{D W}$ on these MK lattices. Indeed, in this limit, $P\left(E_{p}, E_{a}\right)$ becomes Gaussian and so one can perform a cumulant expansion about this Gaussian, leading to a $1 / b$ series. This scheme extends the work of Southern and Young [13] who assumed that $P\left(E_{p}, E_{a}\right)$ was Gaussian even for finite $b$. For general $\ell$ and $b$ we obtain

$$
\theta_{D W}(\ell, b)=\frac{d}{2}+a_{0}(\ell)+\frac{a_{1}(\ell)}{b}+O\left(\frac{1}{b^{2}}\right) .
$$

When $\ell=2$, we find $a_{0}=-1.2302$ and $a_{1}=-0.04573$ (see the appendix for a derivation). In Fig. [5 we plot the difference between the numerically obtained $\theta_{D W}(\ell=$ $2, b)$ and the terms of the expansion given in Eq. 8 . This allows us to determine numerically the next term of the expansion, and we find $-0.045 \pm 0.001 / b^{2}$. This value could be obtained analytically, but we have not pushed the analytical calculation to that order. Note that the $1 / b$ expansion corresponds to an expansion in $e^{-d}$ where $d$ is the dimension of space. This justifies the fact that the $1 / b$ expansion is quite accurate all the way down to $d=3$ (which corresponds to $b=4$ ). Finally, when $\ell \rightarrow \infty$, we obtain $a_{0}=3 / 2$. These results show that $\Theta_{s}=\theta_{D W}<$ $\Theta_{f}$, justifying the neglect of the "correction" terms in section IVA from which we concluded that $\Theta_{f}=d / 2$.

Before going on to the mean field case, let us remark that the MK value for $\theta_{D W}$ is quite close the actual value in the EA model. If we use the standard choice for $d=3, \ell=2$ with $b=4$, the MK prediction is $\theta_{D W} \approx 0.255$. One can also use the choice $\ell=3$ with $b=9$ for which $\theta_{D W} \approx 0.242$. These values are to be compared to current estimates for $\theta_{D W}$ in the EA model, $\theta_{D W}=0.21 \pm 0.02[23]$ and $\theta_{D W}=0.19 \pm 0.02$ 24]. A similarly good comparison occurs when $d=2$.

\section{B. Mean-field models}

We have seen that the prediction of the MK approach is $\Theta_{s}=\theta_{D W}$; in $d=3$, this gives either $\Theta_{s}=0.25$ (the MK value) or $\Theta_{s} \approx 0.20$ (if one uses the $d=3$ EA model values for $\left.\theta_{D W}\right)$. In the case of the mean field models, there is no way to introduce domain walls, and so we simply focus on their prediction for $\Theta_{s}$.

Consider first the finite size effects in the SK model. Parisi et al. 25] have computed analytically how various quantities converge to their large $N$ limit. An $N^{-2 / 3}$ convergence is the general rule, though for the energy density they were able to compute the finite size correction only at the critical temperature $T_{c}$ and on the de Almeida-Thouless line. Nevertheless, the natural extrapolation is that this law should apply to all $T \leq T_{c}$, leading to the prediction $\Theta_{s} / d=1 / 3$. (Note that this prediction is very different from that of [18] for which $\Theta_{s} / d=\Theta_{f} / d=1 / 6$.) To our knowledge, the possibility that $\Theta_{s} / d=1 / 3$ for the SK ground state energy was first brought up by Palassini [26] in his numerical studies. We can extend his analysis with our data; identifying as before $L$ with $N^{1 / d}$, we perform fits of the mean ground state energy to Eq. 1. We then find $\Theta_{s} / d=0.34 \pm 0.02$, in complete compatibility with $1 / 3$; note that when plotted as a function of $N^{-2 / 3}$, the data is very linear starting from $N=50$ (see the inset of Fig. 6). In addition, if we perform the fit while forcing $\Theta_{s} / d=1 / 3$, we find that the ground state energy density at $N=\infty$ is $e_{0}=-0.7637 \pm 0.0002$, in very good agreement with the exact [1] result $e_{0}=-0.7633 \ldots$

In contrast to what happens for $\Theta_{f} / d, \Theta_{s} / d$ is the same in the SK model and in the fixed connectivity models we have considered; this is illustrated in Fig. [6] For instance, for connectivity 6 , a power law fit gives $\Theta_{s} / d=0.35 \pm 0.03$ with $\chi^{2}=9.7$ for 7 degrees of freedom. In fact, the value $\Theta_{s} / d=1 / 3$ works very well for all four connectivities we studied, and we are tempted to consider that this value is the exact exponent. The same conclusion was reached by Boettcher [27]. 


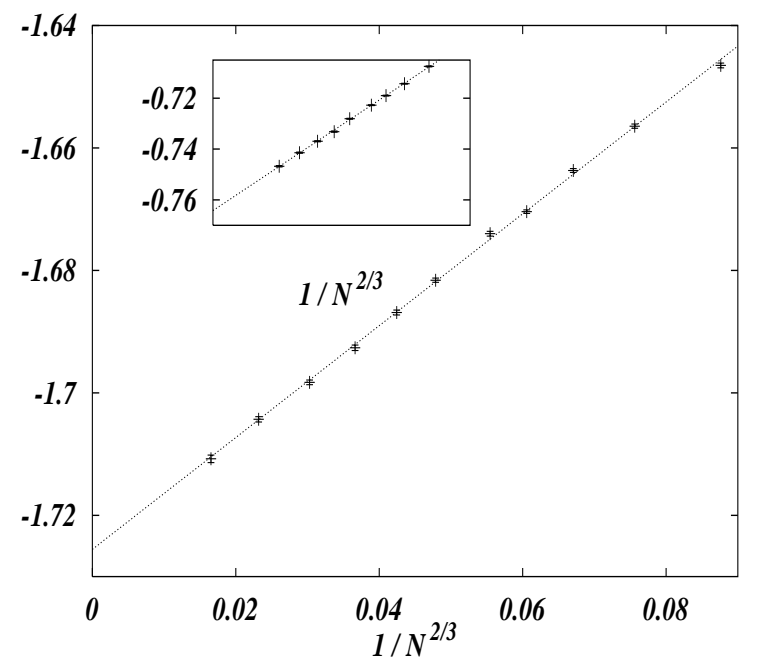

FIG. 6: Mean ground state energy density versus $N^{-2 / 3}$ for the diluted mean field model with connectivity 6 and for the SK model (inset). Error bars are included.

\section{Edwards-Anderson models}

Now it is time to compare the MK and mean field "predictions" to our measurements of $\Theta_{s}$ in the EA models. Let us begin with the case $d=2$. We fit our $4 \leq L \leq 10$ data to Eq. 1 where $e_{0}, e_{1}$ and $\Theta_{s}$ are ajustable parameters; that gives us the estimate $\Theta_{s}=-0.35 \pm 0.01$. (The associated $\chi^{2}$ is 1.9 for 5 degrees of freedom.) The resulting fit is displayed along with the data in the inset of Fig. 17 Given our statistical error and perhaps more importantly the systematic effects associated with $L$ not being very large, this value is reasonably compatible with the MK prediction $\Theta_{s}=\theta_{D W}$ since $\theta_{D W}=-0.28$ [28, 29, 30].

A further argument in favor of $\Theta_{s}=\theta_{D W}$ is as follows. It is known that whether one modifies the boundary conditions [31] or increases the size of the system [32], the fractal dimension of the surface of the clusters of spins that change is approximately the same. It is thus likely that the same type of excitations are involved in determining $\Theta_{s}$ and $\theta_{D W}$. As a consequence, we believe that the $d=2$ EA model is described by a scaling theory with $\theta_{s}=\theta_{D W}$, but also with $\Theta_{f} \neq \theta_{D W}$ of course. Note that the mean field prediction $\left(\Theta_{s}=2 / 3\right)$ is clearly off; however, one cannot appeal to mean field when $d=2$ because one is below the lower critical dimension.

Let us now move on to the $d=3$ EA model which is more challenging and has a spin glass transition at $T_{c}>0$. We use the same fitting function (Eq. 1) as before; the best fit then gives a good $\chi^{2}$ and a mean ground state energy growing as $-1.700 L^{3}+1.9 L^{\Theta_{s}}$ with $\Theta_{s}=0.10 \pm 0.03$. This fit is displayed in the main part of Fig. 17 However this value of $\Theta_{s}$ easily changes by 0.1 when removing some of the data points, and in fact the fit sometimes even leads to negative values for $\Theta_{s}$. Thus

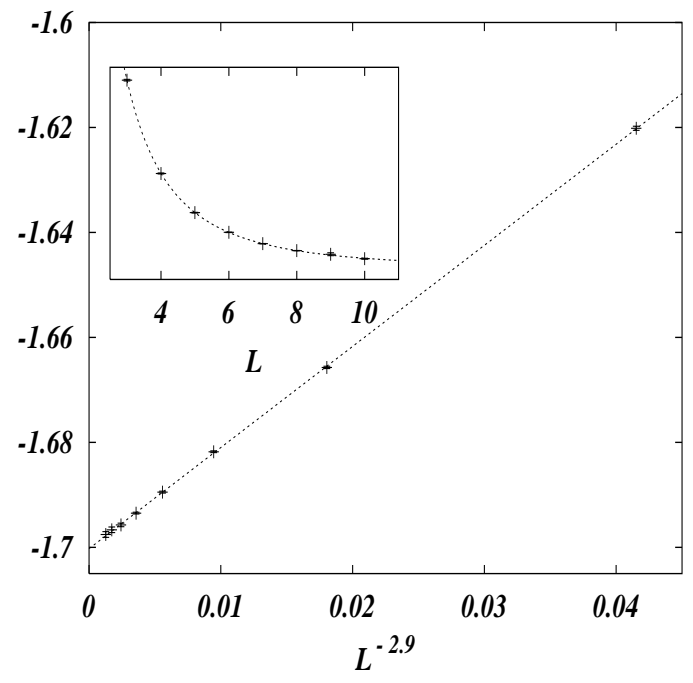

FIG. 7: Mean ground state energy density and the best fits for the $d=2$ (inset) and $d=3 \mathrm{EA}$ spin glass with Gaussian couplings. We find $\Theta_{s}(d=2) \approx-0.35$ and $\Theta_{s}(d=3) \approx 0.1$. These value are "close" to $\theta_{D W}$, suggesting $\Theta_{s}=\theta_{D W}$. Error bars are included.

at best we can say that $\Theta_{s}$ is small, somewhere between 0.0 and 0.2 . One can compare this result to "theory". The mean field value $\left(\Theta_{s}=1.0\right)$ is completely ruled out, whereas the MK prediction $\Theta_{s}=\theta_{D W}$ is quite acceptable since $\theta_{D W} \approx 0.2[23,24]$. However, another possibility is that the discrepancy we find has a physical origin and that in fact $\Theta_{s} \neq \theta_{D W}$. Since this issue is important, we push the analysis a bit further as follows. Given a putative value for $\Theta_{s}$, we adjust $e_{0}$ so that the plot of $\overline{E_{J}(L)}-e_{0} L^{3}$ versus $L^{\Theta_{s}}$ passes through the origin. For $\Theta_{s}$ outside the range $[0.05,0.2]$, the data has visible curvature. In the inset of Fig. [8] we show $\overline{E_{J}(L)}-e_{0} L^{3}$ as a function of $L$; we see that there is no clear trend, so even $\Theta_{s}=0$ seems possible. Such a value could be interpreted from the (mean-field-like) behavior of system-size excitations found in this model 33, 34]. The analogous analysis in the $d=2$ case is shown in the main part of Fig. 8 when we use the value $\Theta_{s}=-0.28$; there the expected corrections to scaling work quite well.

Of course it would be useful to have a significantly smaller error bar for $\Theta_{s}$ but we cannot go much beyond what we have done here: the statistical error on $\overline{E_{J}}$ grows as $L^{d / 2}$, and the amount of computation time grows still faster, so we cannot obtain useful information at large $L$.

We have no data for $d=4$, but let us briefly consider the published work by Hartmann [35] where he used the $J_{i j}= \pm 1 \mathrm{EA}$ model. (One expects its $\Theta_{s}$ to be the same as in the Gaussian case.) Analyzing his values for the mean ground state energy for $2 \leq L \leq 7$, we find $\Theta_{s}=$ $0.2 \pm 0.1$; this does not compare well with his estimate $\theta_{D W}=0.65 \pm 0.04$. But, if we remove the $L=2$ point from the fit, we find $\Theta_{s}=0.7 \pm 0.2$ which is in good agreement with $\Theta_{s}=\theta_{D W}$. We have also analyzed the 


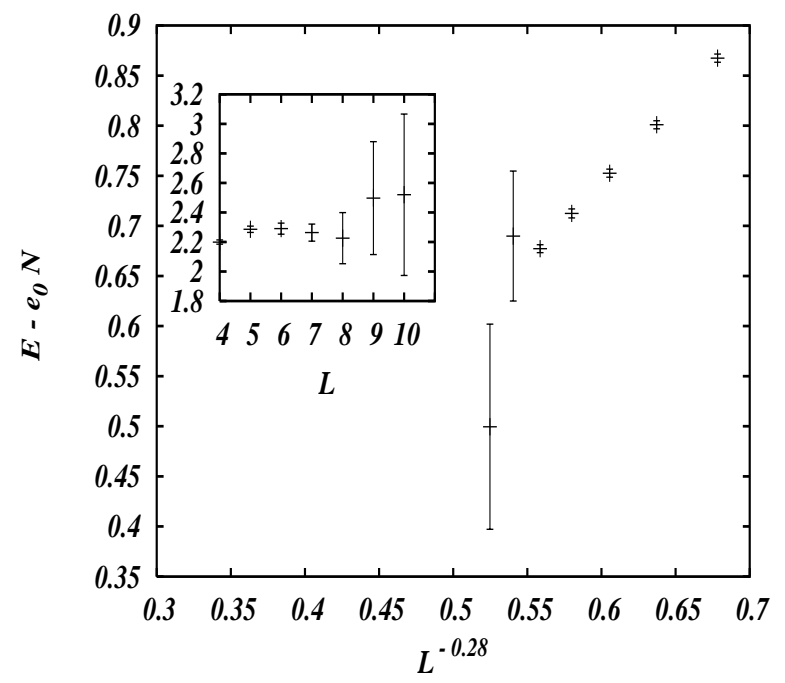

FIG. 8: $\overline{E_{J}(L)}-e_{0} L^{2}$ versus $L^{-0.28}$ (main figure) and $\overline{E_{J}(L)}-$ $e_{0} L^{3}$ versus $L$ (inset) for the $d=2(d=3)$ EA spin glass with Gaussian couplings.

data of Boettcher and Percus [36], and this leads to the same conclusion. In summary, we cannot exclude that $\Theta_{s}<\theta_{D W}$, but the MK prediction $\Theta_{s}=\theta_{D W}$ works surprizingly well in the finite dimensional EA models. On the contrary, the mean field prediction is definitely off, and that of the Mattis spin glass is completely wrong since it gives $\Theta_{s}=-\infty$.

\section{CASE OF $+/-J$ COUPLINGS}

It is widely believed that exponents are universal, i.e., independent of the detailed microscopic nature of the disorder. (Note however that there are longstanding claims of universality violations in spin glasses [37].) In particular, for the $d=3 \mathrm{EA}$, numerical computations of $\theta_{D W}$ confirm this to a large extent: one has $\theta_{D W}=0.21 \pm 0.02$ for the Gaussian 23 and $\theta_{D W}=0.19 \pm 0.02$ for the $J_{i j}= \pm 1$ [24] models. However, if $T_{c}=0$ as arises in $d=2$, one may expect several universality classes and thus some influence of the microscopic properties (i.e., the distribution of the $J_{i j}$ ) on the macroscopic properties (e.g., exponents). Note first that $\theta_{D W}$ is known to be different for Gaussian and binary $\left(J_{i j}= \pm 1\right)$ couplings [29] in the $d=2 \mathrm{EA}$ model. A posteriori, that is not so surprising since the associated quantities (for instance domain wall energies) go to zero rather than to infinity; they can thus easily be affected by microscopic details. This issue can be investigated within the framework of MK lattices. We find identical values of $\theta_{D W}$ in the Gaussian and the $J= \pm 1$ cases whenever $\theta_{D W}>0$. However in $d=2$ and using $b=\ell=2$, the Gaussian model gives $\theta_{D W}=-0.22$; on the contrary, the $J_{i j}= \pm 1$ case leads to $\theta_{D W}=-\infty$, meaning that the domainwall energies decrease exponentially with the size of the

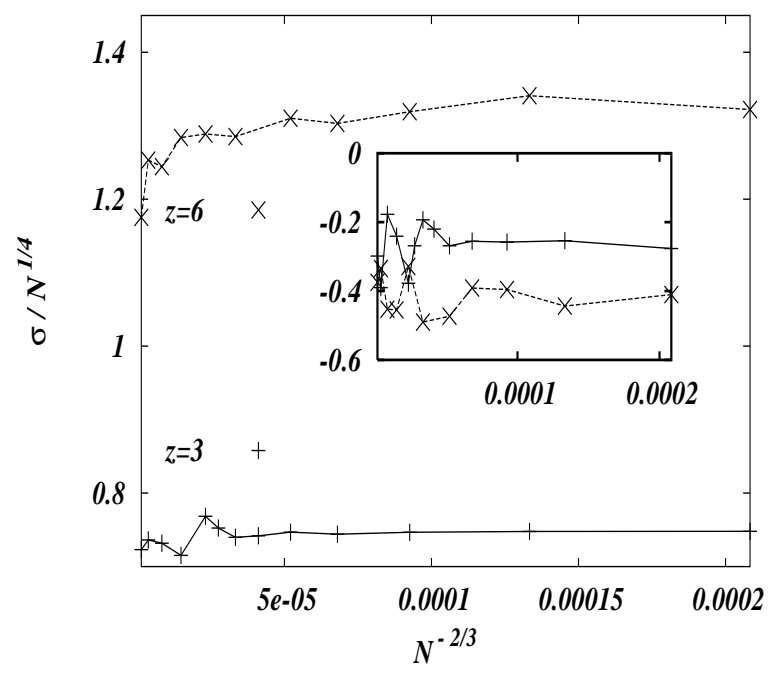

FIG. 9: Standard deviation of $E_{J}$ divided by $N^{1 / 4}$ for the $z=$ 3 and 6 fixed connectivity models, but with binary couplings, $J_{i j}= \pm 1$. Inset: skewness of the distribution of the ground state energy for these same two models.

system rather than as a power law. Thus either there is violation of universality (which seems unlikely to us) or there are several universality classes when $\theta_{D W} \leq 0$; Amoruso et al. [38] have given evidence in favor of the latter possibility since the first version of this paper was posted.

Given these amendments to the scope of universality, the microscopic details should indeed be irrelevant for quantities associated with diverging energy scales. The surprising claim we bring forward here is that this expectation is still too strong: a counter example is provided by $\Theta_{f}$ in the mean field fixed connectivity graphs. Indeed, we saw in the case of Gaussian couplings that $\Theta_{f} / d=1 / 2$. Now in Fig. [ we show the rescaled standard deviation of $E_{J}$ for the case where $J_{i j}= \pm 1$ on fixed connectivity graphs; the standard deviations have been divided by $N^{1 / 4}$. If $\Theta_{f} / d=1 / 2$, we should see a rapid divergence of the plotted values with increasing $N$, but instead the curves are relatively flat and decreasing! In fact, when performing fits, we find that $\Theta_{f} / d$ is between $1 / 4$ and $1 / 5$.

To give further evidence that the Gaussian and $J_{i j}=$ \pm 1 cases scale differently, we plot in the inset of this figure the values of the skewness of the distribution of $E_{J}$ using the same models and values of $N$ as in the main figure. Although our data is noisy, the skewness shows no sign of going to zero when $N \rightarrow \infty$. Taken at face value, this means that the distribution of $E_{J}$ is not Gaussian in the large $N$ limit when $J_{i j}= \pm 1$, in sharp contrast to what happens in the case of Gaussian couplings. To drive this point home further, we find that as $z$ increases, the skewness and kurtosis grow in magnitude and seem to approach the values we find in the SK. (But as before, our values become imprecise at large $N$.) We thus conjecture 
that each $z$ is associated with a different universality class and that, as $z \rightarrow \infty$, one converges to the class to which the SK model belongs. One way to justify this is to consider that in these models $z$ is related to dimension rather than to lattice connectivity; the universality class will then change with $z$. To a large extent, all different trends give further credence to the claim that $\Theta_{f} / d=1 / 4$ in the SK model if that is the correct value for the fixed connectivity models with binary couplings.

How can one understand this "break-down" of universality when the underlying energies diverge? Recall that for the $J_{i j}= \pm 1$ spin glass model on a fixed connectivity graph, the local environment of a spin has no disorder out to finite distances: any sign of the $J_{i j}$ can be gauged away so that all the sample-dependent fluctuations arise "at infinity". On the contrary, in the Gaussian case the $J_{i j}$ fluctuations are local, leading to $O\left(N^{1 / 2}\right)$ fluctuations in the total energy. Taken at face value, this argument also applies to coordination fluctuations; if this is true, the \pm 1 Viana-Bray model [39] will have $\Theta_{f}=d / 2$.

To summarize, the exponent $\Theta_{f}$ depends on the details of the underlying $J_{i j}$ distribution even though the energy scale (of fluctuations) diverges. Not surprisingly, we also find that $P_{N}\left(E_{J}\right)$ for that system does not become Gaussian. Finally, in spite of this major change of behavior when going from Gaussian $J_{i j}$ to binary values, we find that $\Theta_{s} / d=1 / 3$ very precisely in both cases.

\section{DISCUSSION AND CONCLUSION}

Let us go over the main results of the present work on spin glasses. First, the sample to sample fluctuations of the ground state energy grow as the square-root of the volume in almost all models so the fluctuation exponent satisfies $\Theta_{f}=d / 2$. Furthermore, the distribution over disorder of the ground state energy probably tends towards a Gaussian in the large volume limit as suggested by Wehr and Aizenman [11]. There are two notable exceptions to this picture: the SK model and the fixed connectivity mean field models having $J_{i j}= \pm 1$. In those two cases, the sample to sample fluctuations are much smaller, and we find $\Theta_{f} \approx d / 4$, even though a still smaller value cannot be excluded. (Note that fluctuations which are smaller than square root of the volume $N$ are also a characteristic of the directed polymer in a random medium.) On the other hand, for finite dimensional lattices, one expects the finite density of unfrustrated regions to contribute $O\left(N^{1 / 2}\right)$ fluctuations to the ground state energy. A trivial example where this is the case is the (unfrustrated) Mattis model; there the variance of the ground state energy is obviously maximal and equal to $z N\left[\left\langle J^{2}\right\rangle-\langle J\rangle^{2}\right] / 2$ for a connectivity $z$.

We also studied the exponent $\Theta_{s}$ giving the corrections to the scaling of the average (extensive) ground state energy. For the $d=2$ and 3 EA models, we find that the equality $\Theta_{s}=\theta_{D W}$ holds within our limited precision. This means that corrections to scaling are associ- ated with domain walls. However, we were not able to rule out $\Theta_{s}=0$ in $d=3$, leaving the door open to other interpretations. In the context of the MK lattices, the equality $\Theta_{s}=\theta_{D W}$ holds exactly. Physically, this equality corresponds to the fact that in a finite fraction of the samples, the boundary conditions force a domain wall "defect" to be present in the ground state. (Note that the finite size corrections are indeed always positive for fixed and periodic boundary conditions). Furthermore, we were able to compute $\theta_{D W}$ analytically to order $1 / b$ when the dimension becomes large. In the case of the mean field models (SK or diluted graphs), the situation is quite different: the corrections to scaling grow as $N^{1 / 3}$. Since one expects $\theta_{D W} \sim d / 2$ in large dimensions, the result $\Theta_{s} / d=1 / 3$ for these mean field models differs from the large dimension limit of $\theta_{D W} / d$.

Finally, we have exhibited examples where the exponent $\Theta_{f}$ depends on the distribution of the $J_{i j}$, even though energy fluctuations diverge with size, i.e., $\Theta_{f}>0$.

Our work suggests several paths for further studies. (a) Can one establish the value of $\Theta_{f} / d$ for the SK model? (b) Since large fluctuations of order $N^{1 / 2}$ are detrimental in a numerical determination of the average ground state energy, is there a way to substract a (computable) contribution of satisfied bonds so as to reduce the variance? (c) Is $\Theta_{s}=\theta_{D W}$ in finite dimensional spin glasses or is $\Theta_{s}$ smaller? This second possibility could follow from other types of excitations whose exponents are smaller than $\theta_{D W}$.

Acknowledgments - We thank A. Billoire, K. Binder, D. S. Fisher, M. Mézard, M. Palassini, G. Parisi, R. da Silveira and O. White for stimulating discussions. This work was supported in part by the European Community's Human Potential Programme under the contracts HPRN-CT-2002-00307 and HPRN-CT2002-00319. F.K. acknowledges financial support from the MENRT. The LPTMS is an Unité de Recherche de l'Université Paris XI associée au CNRS.

\section{Appendix: Exponents in the Migdal-Kadanoff lattices and the large dimensional limit}

In this appendix, we focus on the simplest case $\ell=2$. To write a recursion relation, one needs to keep track of two energies, $E_{p}^{(r)}$ and $E_{a}^{(r)}$ that give the ground state energy of the MK lattice at the $r$-th application of the recursion when the two exterior spins are respectively parallel and antiparallel. For a $b$-branch lattice with $\ell=$ 2 , the ground state energies at the $(r+1)$-th application of the recursion read:

$$
\begin{array}{r}
E_{p}^{(r+1)}=\sum_{\alpha=1}^{b} \min \\
\left(E_{p}^{(r)}(1, \alpha)+E_{p}^{(r)}(2, \alpha), E_{a}^{(r)}(1, \alpha)+E_{a}^{(r)}(2, \alpha)\right)
\end{array}
$$




$$
\begin{array}{r}
E_{a}^{(r+1)}=\sum_{\alpha=1}^{b} \min \\
\left(E_{p}^{(r)}(1, \alpha)+E_{a}^{(r)}(2, \alpha), E_{a}^{(r)}(1, \alpha)+E_{p}^{(r)}(2, \alpha)\right)
\end{array}
$$

where the index 1, 2 refers to the two bonds in the $\ell$ direction. This equation says that along all the $b$ branches, one has to choose the orientation of the middle spin (that is decimated) such that the energy contribution is minimized, given the orientations of the external spins. Note that all the random variables that appear in this equation are independent as soon as they live on different bonds.

If one assumes that the distribution $P^{(r)}\left(E_{p}, E_{a}\right)$ of energies at the $r$-th generation can be written for large $r$ in the scaling form

$$
P^{(r)}\left(E_{p}, E_{a}\right)=1 / \sigma_{r}^{2} f\left(\left[E_{p}-\mathcal{E}_{r}\right] / \sigma_{r},\left[E_{a}-\mathcal{E}_{r}\right] / \sigma_{r}\right)
$$

then it is immediate to show (using the independence of the branches) that $\mathcal{E}_{r+1}=(2 b) \mathcal{E}_{r}$ and $\sigma_{r+1}=\sqrt{2 b} \sigma_{r}$, so that $\mathcal{E}_{r} \sim(2 b)^{r}$ and $\sigma_{r} \sim(2 b)^{r / 2}$. Since the number of spins is given by $(2 b)^{r}$, one immediately finds that the ground state energy is extensive and that the fluctuations are described by $\Theta_{f} / d=1 / 2$.

Define $\Delta^{(r)}$ as $E_{p}^{(r)}-E_{a}^{(r)}$. It is easy to show that $\Delta^{(r)}$ obeys an autonomous recursion relation [13]:

$$
\Delta^{(r+1)}=\sum_{\alpha=1}^{b} \epsilon(\alpha) \min \left(\left|\Delta^{(r)}(1, \alpha)\right|,\left|\Delta^{(r)}(2, \alpha)\right|\right),
$$

where $\alpha$ labels branches and

$$
\epsilon(\alpha)=-\operatorname{sign}[\Delta(1, \alpha)] \operatorname{sign}[\Delta(2, \alpha)] .
$$

Using the independence of the $\Delta$ 's corresponding to different branches, one finds that $\Sigma_{r}$, the RMS of the distribution of the $\Delta$ 's, obeys the following equation:

$\Sigma_{r+1}^{2}=4 b \Sigma_{r}^{2} \int_{0}^{\infty} \mathrm{d} x g(x)\left[\int_{0}^{x} \mathrm{~d} y y^{2} g(y)+x^{2} \int_{x}^{\infty} \mathrm{d} y g(y)\right]$,

where $g($.$) is the distribution of \Delta / \Sigma$ that is independent of $r$ for large $r$. From this relation, one finds that $\Sigma_{r} \sim \lambda^{r}$ with

$$
\lambda^{2}=4 b \int_{0}^{\infty} \mathrm{d} x g(x)\left[\int_{0}^{x} \mathrm{~d} y y^{2} g(y)+x^{2} \int_{x}^{\infty} \mathrm{d} y g(y)\right]
$$

where of course $g($.$) depends on b$. The energy scale for flipping the relative sign of the exterior spins is $\Sigma$ and so the exponent $\theta_{D W}$ is given by

$$
\theta_{D W}=\frac{\ln \lambda}{\ln 2}
$$

In the large dimension limit, for which $b \rightarrow \infty$, it is clear that, using the central limit theorem, the distribution of $\Delta$ is Gaussian. Since $g($.$) is then known, \lambda$ can be computed from Eq. 15 giving

$$
\lambda^{2}=0.36338 b,
$$

a result first obtained by Southern and Young.

When $b$ is large but not infinite, the first correction to the Gaussian is of order $1 / b$ (because the $\Delta$ have a symmetric distribution) and reads:

$$
g(x)=\frac{1}{\sqrt{2 \pi}}\left[1+\frac{\kappa}{24 b} \frac{\partial^{4}}{\partial x^{4}}+\ldots\right] e^{-x^{2} / 2}
$$

where $\kappa$ is the kurtosis of the initial variable, i.e., $\epsilon \min (|\Delta(1)|,|\Delta(2)|)$. To first order in $1 / b$, this kurtosis can be computed by assuming that the $\Delta$ are Gaussian, and one finds $\kappa=0.434215$. Injecting the expression of $g(x)$ in Eq. (15) then gives $\lambda$ to order $1 / b$ : $\lambda^{2}=0.36338 b-0.023035$, and finally the result given in the main text after Eq. (8).

This calculation can be extended to next order: $\kappa$ will acquire a $1 / b$ contribution and there will be corrections to $g(x)$ of order $1 / b^{2}$ coming from the non zero sixth cumulant of $\epsilon \min (|\Delta(1)|,|\Delta(2)|)$, computed as if the $\Delta$ 's were Gaussian. One could also, with more work, compute $\lambda$ for $\ell \neq 2$. In the limit $\ell \rightarrow \infty$, the problem becomes soluble again using the theory of extreme value statistics for handling the variable $\min (|\Delta(1)|,|\Delta(2)|, \ldots,|\Delta(\ell)|)$.
[1] M. Mézard, G. Parisi, and M. A. Virasoro, Spin-Glass Theory and Beyond, Vol. 9 of Lecture Notes in Physics (World Scientific, Singapore, 1987).

[2] Spin Glasses and Random Fields, edited by A. P. Young (World Scientific, Singapore, 1998).

[3] J. Galambos, The asymptotic Theory of Extreme Order Statistics (R.E. Krieger Publishing Co., Malabar, Florida, 1987).

[4] D. Carpentier and P. Le Doussal, Phys. Rev. E 63, 026110 (2001).

[5] B. Derrida, Phys. Rev. B 24, 2613 (1981).

[6] J.-P. Bouchaud and M. Mézard, J. Phys. A 30, 7997
(1997).

[7] T. Halpin-Healy and Y. C. Zhang, Physics Reports 254, 215 (1995).

[8] W. L. McMillan, Phys. Rev. B 29, 4026 (1984).

[9] A. J. Bray and M. A. Moore, in Heidelberg Colloquium on Glassy Dynamics, Vol. 275 of Lecture Notes in Physics, edited by J. L. van Hemmen and I. Morgenstern (Springer, Berlin, 1986), pp. 121-153.

[10] D. S. Fisher and D. A. Huse, Phys. Rev. Lett. 56, 1601 (1986).

[11] J. Wehr and M. Aizenman, J. Stat. Phys. 60, 287 (1990).

[12] S. F. Edwards and P. W. Anderson, J. Phys. F 5, 965 
(1975).

[13] B. W. Southern and A. P. Young, J. Phys. C 10, 2179 (1977).

[14] D. Sherrington and S. Kirkpatrick, Phys. Rev. Lett. 35, 1792 (1975).

[15] C. de Dominicis and Y. Goldschmidt, J. Phys. A Lett. 22, L775 (1989).

[16] J. Houdayer and O. C. Martin, Phys. Rev. E 64, 056704 (2001), cond-mat/0105617.

[17] I. Kondor, J. Phys. A 16, L127 (1983).

[18] A. Crisanti, G. Paladin, J.-J. Sommers, and A. Vulpiani, J. Physique I France 2, 1325 (1992).

[19] T. Aspelmeier, M. A. Moore, and A. P. Young, Phys. Rev. Lett. 90, 127202 (2003), cond-mat/0209290.

[20] S. Cabasino, E. Marinari, P. Paolucci, and G. Parisi, J. Phys. A 21, 4201 (1988).

[21] M. Palassini, private communication.

[22] T. Aspelmeier and M. Moore, Phys. Rev. Lett. 90, 177201 (2003), cond-mat/0211707.

[23] M. Palassini and A. P. Young, J. Phys. Soc. Jpn. 69, 165 (2000), cond-mat/9910278.

[24] A. K. Hartmann, Phys. Rev. E 59, 84 (1999).

[25] G. Parisi, F. Ritort, and F. Slanina, J. Phys. A 26, 3775 (1993).

[26] M. Palassini, Ph.D. thesis, Scuola Normale Superiore,
Pisa, Italy, 2000.

[27] S. Boettcher, Eur. Phys. J. B 31, 29 (2003), condmat/0208196.

[28] H. Rieger et al., J. Phys. A 29, 3939 (1996).

[29] A. K. Hartmann and A. P. Young, Phys. Rev. B 64, 180404 (2001), cond-mat/0107308.

[30] A. C. Carter, A. J. Bray, and M. A. Moore, Phys. Rev. Lett 88, 077201 (2002).

[31] M. Palassini and A. P. Young, Phys. Rev. Lett. 83, 5126 (1999), cond-mat/9906323.

[32] A. Middleton, Phys. Rev. Lett. 83, 1672 (1999).

[33] F. Krzakala and O. C. Martin, Phys. Rev. Lett. 85, 3013 (2000), cond-mat/0002055.

[34] M. Palassini and A. P. Young, Phys. Rev. Lett. 85, 3017 (2000), cond-mat/0002134.

[35] A. K. Hartmann, Phys. Rev. E 60, 5135 (1999), condmat/9904296.

[36] S. Boettcher and A. Percus, Phys. Rev. Lett. 86, 5211 (2001), cond-mat/0010337.

[37] P. Mari and I. Campbell, Phys. Rev. E, 59, 2653 (1999).

[38] C. Amoruso, E. Marinari, O. C. Martin, and A. Pagnani, Phys. Rev. Lett. 91, 087201 (2003), cond-mat/0305042.

[39] L. Viana and A. J. Bray, J. Phys. C 18, 3037 (1985). 